\title{
Generación y representación de Indicadores de calidad de aire: caso de estudio aplicado a Manizales
}

\section{Generation and representation of air quality indexes: a case study applied to Manizales}

\author{
Jade Alexandra Li-Ramírez \\ M.Sc. (c) Ing. Química \\ Universidad Nacional de Colombia \\ Manizales, Colombia \\ jalir@unal.edu.co \\ Néstor Darío Duque-Méndez \\ Ph.D. Ingeniería \\ Universidad Nacional de Colombia \\ Manizales, Colombia \\ ndduqueme@unal.edu.co
}

\author{
Ángela María Pérez-Zapata \\ Admon. de Sistemas Informáticos (c) \\ Universidad Nacional de Colombia \\ Manizales, Colombia \\ amperezz@unal.edu.co \\ Beatriz Helena Aristizábal-Zuluaga \\ Ph. D. Ciencias Químicas \\ Universidad Nacional de Colombia \\ Manizales, Colombia \\ bharistizabalz@unal.edu.co
}

\begin{abstract}
Resumen-Se implementaron indicadores de calidad del aire para el dominio de Manizales y se estructuró una bodega de datos que permite a la población visualizar y obtener esta información gratuitamente. Dos tipos de indicadores fueron calculados: índices de calidad del aire (ICA) y porcentaje excedencia de los límites normados (PELN). Se analizaron e interpretaron estos indicadores para los contaminantes Dióxido de azufre $\left(\mathrm{SO}_{2}\right)$, Ozono $\left(\mathrm{O}_{3}\right)$ y Material particulado $\left(\mathrm{PM}_{10}\right.$ y $\left.\mathbf{P M}_{2.5}\right)$, utilizando el software $\mathbf{R}$ y la librería Openair. La bodega de datos puede ser visualizada desde la plataforma virtual llamada Centro de Datos e Indicadores Ambientales de Caldas (CDIAC), el cual cumple con la función de almacenar, procesar y poner a disposición del público la información obtenida desde las estaciones de monitoreo del Sistema de Vigilancia de Calidad del Aire de Manizales. Los resultados mostraron bajas concentraciones de los contaminantes analizados con valores de ICA normalmente entre 10 y 60 para material particulado, y entre 0 y 27 para los gases. En general los ICA se ubicaron en la categoría Buena y en pocas ocasiones en la categoría Moderada. EI PELN de los contaminantes no ha sobrepasado el $1 \%$.
\end{abstract}

Palabras clave—Contaminación atmosférica, índice de calidad del aire, bodega de datos, Manizales.

Abstract- Air quality indexes for Manizales domain were implemented and a data warehouse was structured, allowing to people to display and obtain indexes information for free. Two kinds of indexes were calculated: Air Quality Indexes (AQI) and Percentage of exceedance of normed Limits (PELN). Indexes for Sulfur dioxide $\left(\mathrm{SO}_{2}\right)$, Ozone $\left(\mathrm{O}_{3}\right)$ and Particulate matter $\left(\mathrm{PM}_{10}\right.$ and $\left.\mathrm{PM}_{2.5}\right)$ were calculated and analyzed using $R$ software and Openair library. Access to the data warehouse is possible from the virtual platform called Centro de Datos e Indicadores Ambientales de Caldas (CDIAC) in charge of storing, processing and providing to people the information from the system of air quality monitoring stations. Results exhibit low concentrations of analyzed pollutants, with AQI ranging from 10 to 60 for particulate matter and, from 0 to 27 for gases. Generally AQI remained at Good category and occasionally at Moderate category. The PELN of pollutants did not exceed $1 \%$.

Keywords: Air pollution, air quality indexes, data warehouse, Manizales.

\section{INTRODUCCIÓN}

La atmósfera es una mezcla de gases y aerosoles [1]. La creciente presencia de sustancias contaminantes en la atmósfera producto de diferentes procesos naturales y antropogénicos, está asociada con efectos negativos en el entorno y, principalmente, en la salud humana. Los contaminantes denominados criterio, entre los cuales están el Monóxido de Carbono (CO), Dióxido de Azufre $\left(\mathrm{SO}_{2}\right)$, Ozono $\left(\mathrm{O}_{3}\right)$, y Material particulado $\left(\mathrm{PM}_{10}\right.$ y $\left.\mathrm{PM}_{2.5}\right)$, son regulados con el objetivo de establecer niveles permisibles que protejan el medio ambiente y el bienestar de la población [2]. Según el estudio del Banco Mundial "Prioridades ambientales para la reducción de la pobreza en Colombia", cada año ocurren cerca de 6000 muertes por causa de la contaminación del aire ambiente en Colombia [3]. 
Ante esta situación, tomar medidas correctivas para hacer cumplir la normativa no es suficiente. Esto ha generado la necesidad de realizar mediciones específicas de aquellos contaminantes que afectan en mayor proporción a la población y de informar oportunamente sobre el estado de la calidad del aire, para facilitar a las autoridades ambientales y a los mismos habitantes tomar medidas encaminadas al sostenimiento ambiental y a la protección de la salud pública [4].

Estas mediciones se realizan mediante los Sistemas de Vigilancia de Calidad del Aire (SVCA) constituidos por una red de estaciones de monitoreo que generan cientos de datos diarios correspondientes a las concentraciones medidas de contaminantes criterio [5]. Como complemento a los SVCA se estiman los inventarios de emisiones por fuentes antropogénicas y naturales, los cuales también generan un gran volumen de información. El almacenamiento y procesamiento de este conjunto de datos de forma manual o por métodos convencionales, se convierte en un acto extenuante y que puede generar cierto grado de incertidumbre [6].

Teniendo en cuenta lo anterior, se buscan alternativas para el procesamiento de los datos. La bodega de datos, data warehouse, está orientada al manejo de grandes volúmenes de datos, provenientes de diversas fuentes de información, y que cubren largos periodos de tiempo [7]. Además, brinda mejores formas de administrar la información, disminuyendo la redundancia, es decir, réplicas innecesarias de la información, y agiliza la localización de datos. Las bases de datos están compuestas o permiten la creación de tablas, vectores o contenedores de datos para almacenar información. La estructura de las tablas se define de acuerdo con el propósito de los datos a almacenar, y estas se pueden relacionar entre sí, según lo determine el diseñador o administrador de la base de datos.

El objetivo de esta investigación es actualizar y complementar estudios anteriores [8], generando indicadores de calidad de aire para Manizales, y emplearlos en el análisis de las concentraciones de contaminantes criterio en la ciudad [9]. Por otra parte, se busca representar y publicar los indicadores ICA mediante una herramienta sistemática de cálculo (bodega de datos) que permita el almacenamiento, procesamiento y actualización constante de los datos. Además que permita graficar y obtener tendencias actualizadas según el indicador y el periodo de interés. Se pretende sistematizar la información como base para ayudar a la generación de políticas, estrategias y planes de prevención y regularización de los contaminantes atmosféricos.

\section{DESARROLLO DEL ARTícULO}

La selección de contaminantes para la elaboración de los índices fue basada en la cantidad de información disponible y en su relevancia para el área de estudio. Las mediciones se conocieron a partir de los registros provenientes de las estaciones de monitoreo que abarcan la ciudad. La Tabla I presenta los contaminantes definidos con los que se realizaron los análisis que son $\mathrm{O}_{3}, \mathrm{PM}_{10}$, $\mathrm{PM}_{2.5}, \mathrm{SO}_{2}$ junto con la localización del equipo y la escala temporal para la que se hicieron los cálculos de los indicadores.

TABLA I

CONTAMINANTES MEDIDOS E INFORMACIÓN RELEVANTE DE SUS VARIABLES

\begin{tabular}{|c|c|c|}
\hline Contaminante medido & Localización espacial & Tiempo de los datos \\
\hline Ozono (O3) & Estación Gobernación & $01 / 07 / 2014-01 / 10 / 2015$ \\
\hline Dióxido de azufre (SO2) & Estación Gobernación & $16 / 07 / 2014-01 / 10 / 2015$ \\
\hline & Estación Gobernación & $22 / 04 / 2009-30 / 12 / 2014$ \\
Material particulado menor a & Estación Palogrande & $01 / 09 / 2009-14 / 01 / 2015$ \\
10 micras (PM10) & Estación Nubia & $24 / 10 / 2009-17 / 01 / 2015$ \\
& Estación Liceo & $06 / 01 / 2010-12 / 12 / 2014$ \\
& Estación Milán & $31 / 10 / 2012-30 / 12 / 2014$ \\
\hline Material particulado menor a & Estación Gobernación & $03 / 06 / 2009-30 / 12 / 2014$ \\
2.5 micras (PM2.5) & & \\
\hline
\end{tabular}

Fuente: autores 


\section{1 Área de estudio}

Manizales es la capital del departamento de Caldas. Es una ciudad en el centro occidente de Colombia, ubicada en la Cordillera Central. Se caracteriza por presencia de microclimas y su variabilidad climática en función de la altitud. Según proyecciones del Departamento Administrativo Nacional de Estadística (DANE), para el año 2016 cuenta con una población de 397488 habitantes [10].

Estudios de Manizales revelan que la principal problemática ambiental de la ciudad es la densidad vehicular [11]. Adicionalmente, está ubicada a $28 \mathrm{~km}$ del volcán activo Nevado del Ruiz, lo que se traduce en emisiones de gases y partículas. La calidad del aire de Manizales actualmente se monitorea en cinco (5) puntos de la ciudad, tal como se presenta en la Fig. 1.

\subsection{Obtención de la información}

Los datos brutos y los indicadores son obtenidos desde el Centro de Datos e Indicadores Ambientales de Caldas (CDIAC) [5], una plataforma virtual con una bodega de datos diseñada por los autores de este documento con el fin de consolidar la información ambiental del departamento. La bodega de datos, tiene una estructura llamada "Estrella Descentralizada" como se muestra en la Fig. 2, en esta hay dos tablas denominadas como tablas de hechos y hay tres (3) tablas conocidas como tablas de dimensiones. Este conjunto per- mite realizar búsquedas de los datos desde varios enfoques o bajo diferentes características, a lo que se le denomina granularidad de las búsquedas.

En la bodega de datos se procesa y almacena actualmente datos e indicadores de calidad del aire y meteorológicos. Por medio de esta, la información de calidad del aire puede cruzarse con las variables meteorológicas y así realizar cálculos de los indicadores en condiciones de referencia. Los cálculos se validan, con regularidad, con cálculos realizados manualmente en los software $\mathrm{R}$ y Excel.

Fig. 2. ESTRUCTURA DE LA BODEGA DE DATOS

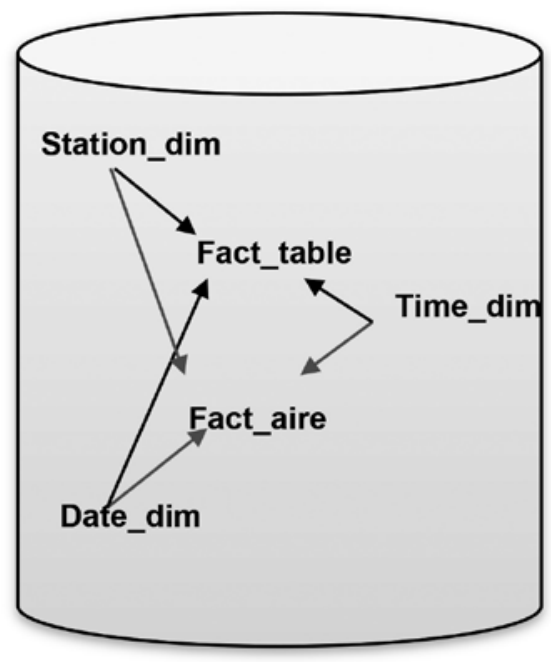

Fuente: autores.

Fig. 1. MAPA DE MANIZALES CON UBICACIÓN GEOGRÁFICA DE ESTACIONES DE CALIDAD DEL AIRE

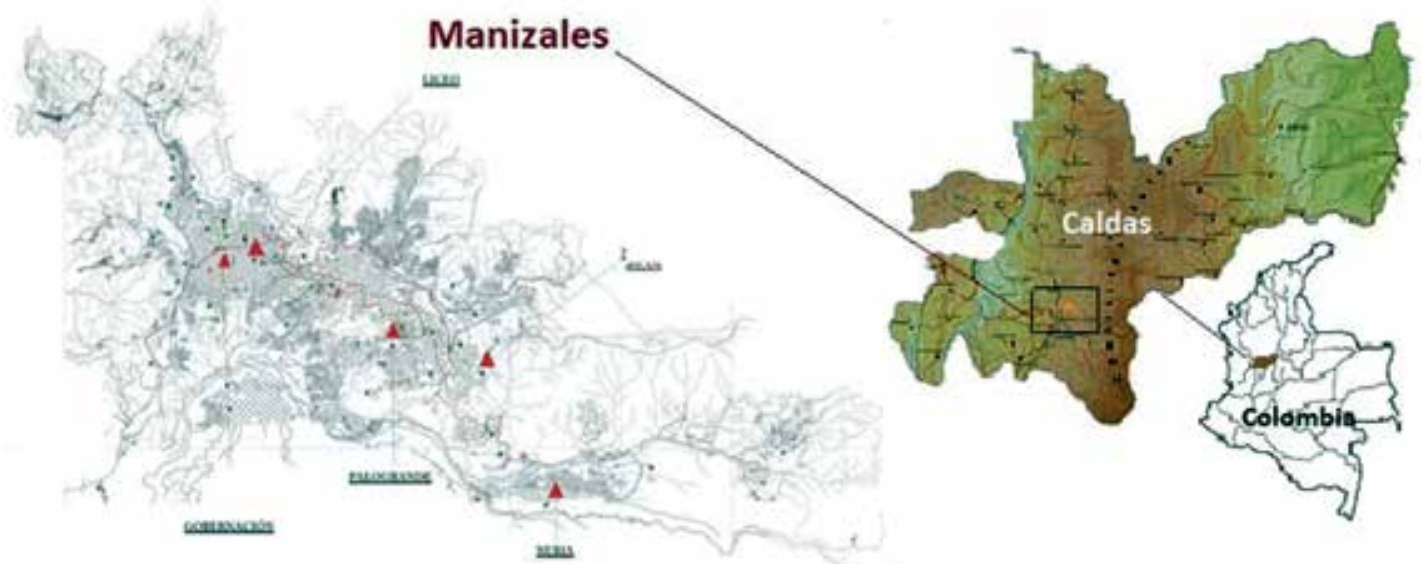




\subsection{Indicadores seleccionados}

Siguiendo los protocolos colombianos, para analizar la información de calidad del aire se escogió determinar y analizar: índices de calidad del aire (ICA) y porcentaje de excedencia de los límites normados (PELN) para los contaminantes seleccionados anteriormente.

\subsection{1 Índices de calidad del aire (ICA)}

Para el cálculo e interpretación de los ICA se utilizó la metodología descrita en el Protocolo para el Monitoreo y Seguimiento de la Calidad del Aire, publicado en 2010 por el Ministerio de Ambiente, Vivienda y Desarrollo Territorial y el Manual de Operación de Sistemas de Vigilancia de Calidad del Aire [12], basado en los límites mostrados en la Tabla II, establecidos por la United States Environmental Protection Agency (EPA). Estos es- tándares permiten establecer el estado de la calidad del aire de una región específica de acuerdo con un rango establecido para cada contaminante y un código de colores [13].

La metodología emplea (1) para el cálculo de Ios ICA:

$$
I_{\text {cont }}=\frac{I_{H i}-I_{L o}}{B P_{H i}-B P_{L o}}\left(C_{\text {cont }}-B P_{L o}\right)+I_{L o}
$$

\section{Donde:}

$\mathrm{I}_{\text {cont }}=$ Índice para el contaminante

$\mathrm{C}_{\text {cont }}=$ Concentración medida para el contaminante $\mathrm{BP}_{\mathrm{Hi}}=$ Punto de corte mayor o igual a $\mathrm{C}_{\text {cont }}$

$\mathrm{BP}_{\mathrm{Lo}}=$ Punto de corte menor o igual a $\mathrm{C}_{\text {cont }}$ $\mathrm{I}_{\mathrm{Hi}}=$ Valor del Índice de Calidad del Aire correspondiente a $\mathrm{BP}_{\mathrm{Hi}}$

ILo = Valor del Índice de Calidad del Aire correspondiente a BPLo

TABLA II

PUNTOS DE CORTE ICA

\begin{tabular}{|c|c|c|c|c|c|c|c|c|c|}
\hline ICA & COLOR & CLASIFICACIÓN & $\begin{array}{l}\mathrm{O}_{3} 8 \mathrm{~h} \\
\mathrm{ppm}\end{array}$ & $\begin{array}{c}0_{3} 1 \mathrm{~h} \\
\mathrm{ppm}(1)\end{array}$ & $\begin{array}{c}\mathrm{PM}_{10} 24 \mathrm{~h} \\
\mu \mathrm{g} / \mathrm{m}^{3}\end{array}$ & $\begin{array}{c}\mathrm{PM}_{2.5} 24 \mathrm{~h} \\
\mu \mathrm{g} / \mathrm{m}^{3}\end{array}$ & $\begin{array}{c}\text { CO 8h } \\
\text { ppm }\end{array}$ & $\begin{array}{l}\mathrm{SO}_{2} 24 \mathrm{~h} \\
\mathrm{ppm}\end{array}$ & $\begin{array}{c}\mathrm{NO}_{2} 1 \mathrm{~h} \\
\mathrm{ppm}\end{array}$ \\
\hline $0-50$ & Verde & Buena & $\begin{array}{l}0.000 \\
0.059\end{array}$ & - & $\begin{array}{c}0 \\
54\end{array}$ & $\begin{array}{c}0.0 \\
15.4\end{array}$ & $\begin{array}{l}0.0 \\
4.4\end{array}$ & $\begin{array}{l}0.000 \\
0.034\end{array}$ & $(2)$ \\
\hline $51-100$ & 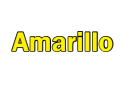 & Moderada & $\begin{array}{l}0.060 \\
0.075\end{array}$ & -- & $\begin{array}{c}55 \\
154\end{array}$ & $\begin{array}{l}15.5 \\
40.4\end{array}$ & $\begin{array}{l}4.5 \\
9.4\end{array}$ & $\begin{array}{l}0.053 \\
0.144\end{array}$ & $(2)$ \\
\hline $101-150$ & Naranja & $\begin{array}{l}\text { Dañina a la salud para } \\
\text { grupos sensibles }\end{array}$ & $\begin{array}{l}0.076 \\
0.095\end{array}$ & $\begin{array}{l}0.125 \\
0.164\end{array}$ & $\begin{array}{l}155 \\
254\end{array}$ & $\begin{array}{l}40.5 \\
65.4\end{array}$ & $\begin{array}{c}9.5 \\
12.4\end{array}$ & $\begin{array}{l}0.145 \\
0.224\end{array}$ & $(2)$ \\
\hline $151-200$ & Rojo & Dañina a la salud & $\begin{array}{l}0.096 \\
0.115\end{array}$ & $\begin{array}{l}0.165 \\
0.204\end{array}$ & $\begin{array}{l}255 \\
354\end{array}$ & $\begin{array}{c}65.5 \\
150.4\end{array}$ & $\begin{array}{l}12.5 \\
15.4\end{array}$ & $\begin{array}{l}0.225 \\
0.304\end{array}$ & $(2)$ \\
\hline $201-300$ & Púrpura & Muy dañina a la salud & $\begin{array}{c}0.116 \\
0.373 \\
(0.155 \\
0.404) \\
(4)\end{array}$ & $\begin{array}{l}0.205 \\
0.404\end{array}$ & $\begin{array}{l}355 \\
424\end{array}$ & $\begin{array}{l}150.5 \\
250.4\end{array}$ & $\begin{array}{l}15.5 \\
30.4\end{array}$ & $\begin{array}{l}0.305 \\
0.604\end{array}$ & $\begin{array}{l}0.65 \\
1.24\end{array}$ \\
\hline $301-500$ & Marrón & Peligrosa & (3) & $\begin{array}{l}0.405 \\
0.604\end{array}$ & $\begin{array}{l}425 \\
604\end{array}$ & $\begin{array}{l}250.5 \\
500.4\end{array}$ & $\begin{array}{l}30.5 \\
50.4\end{array}$ & $\begin{array}{l}0.605 \\
1.004\end{array}$ & $\begin{array}{l}1.25 \\
2.04\end{array}$ \\
\hline
\end{tabular}

Fuente: [12].

(1) Para 03 se calcula el índice usando promedios de 8 horas y de 1 hora.

(2) Para NO2 se tienen en cuenta valores únicamente por encima de 200, debido a que han sido tomados de valores y parámetros EPA.

(3) Valores de concentraciones de 8 horas de ozono no definen valores más altos de ICA (w301). Los valores de ICA de 301 o mayores son calculados con concentraciones de 1 hora de ozono.

(4) Los números entre paréntesis se asocian a valores de 1 hora que se utilizan en esta categoría solo si se superponen. 


\subsubsection{Excedencia de los límites normados (ELN)}

La ELN se realiza calculando promedios aritméticos con las concentraciones medidas por las estaciones de monitoreo entre los intervalos de tiempos definidos en la Tabla III y comparando sus resultados con el nivel máximo legalmente permisible presentado en esta. La tabla es tomada de la Resolución 610 del 2010 del MAVDT [14] que establece la norma de calidad del aire o nivel de inmisión para todo el territorio colombiano en condiciones de referencia para contaminantes criterio [15].

TABLA III

NIVELES MÁXIMOS PERMISIBLES PARA CONTAMINANTES CRITERIO

\begin{tabular}{|c|c|c|}
\hline Contaminante & $\begin{array}{l}\text { Nivel máximo permisible } \\
\left(\mu \mathrm{g} / \mathrm{m}^{3}\right)\end{array}$ & Tiempo de exposición \\
\hline \multirow{2}{*}{ PST } & 100 & Anual \\
\hline & 300 & 24 horas \\
\hline \multirow{2}{*}{ PM10 } & 50 & Anual \\
\hline & 100 & 24 horas \\
\hline \multirow{2}{*}{ PM2.5 } & 25 & Anual \\
\hline & 50 & 24 horas \\
\hline \multirow{3}{*}{ SO2 } & 80 & Anual \\
\hline & 250 & 24 horas \\
\hline & 750 & 3 horas \\
\hline \multirow{3}{*}{ NO2 } & 100 & Anual \\
\hline & 150 & 24 horas \\
\hline & 200 & 1 hora \\
\hline \multirow{2}{*}{03} & 80 & 8 horas \\
\hline & 120 & 1 hora \\
\hline \multirow{2}{*}{$\mathrm{CO}$} & 10000 & 8 horas \\
\hline & 40000 & 1 hora \\
\hline
\end{tabular}

Fuente: [14].

\subsection{Visualización de los indicadores}

Los indicadores se representan en tablas y en gráficas utilizando como plataforma la bodega de datos, donde se descargó la información y se pueden visualizar sus tendencias. Adicionalmente, se utilizó el software R y la librería Openair para mostrar más información de los indicadores. Con estas herramientas se realizaron gráficas, utilizando la media móvil para los datos continuos, es decir, los datos de gases. Para material particulado, se usó el dato diario de concentración que se calcula a partir de las mediciones obtenidas de un equipo High-Vol. Todos los indicadores se determinaron con datos a condiciones estándar.

Se realizaron gráficas de: calendarios, tendencias generales y tendencias por niveles. Cada contaminante se representa por una gráfica diferente de acuerdo con su información.

\section{RESULTADOS}

\subsection{CDIAC - Centro de Datos e Indicadores Ambientales de Caldas}

En las Figuras 3, 4 y 5 se puede observar la plataforma desarrollada a la cual se puede ingresar mediante el link http://cdiac.manizales. unal.edu.co/. Allí es posible visualizar y descargar libremente indicadores ambientales.

La Fig. 3. Muestra la sección en la que se generan los ICA y donde se selecciona el contaminante, la estación de monitoreo y el rango de los años a los que se les desea calcular el indicador.

El resultado de la consulta anterior presenta una tabla como la que se visualiza en la Fig. 4, donde se muestran los resultados del indicador, su clasificación, un análisis de confianza basado en el número de datos con el que se calcula y la desviación estándar con respecto al promedio de la concentración. Esta tabla se puede descargar en formato .csv.

También se puede generar la respectiva gráfica, tal como se muestra en la Fig. 5, que en este caso representa los ICA de $\mathrm{PM}_{2.5}$ para la estación Gobernación, durante el 2014, se observa que también se visualizan en escala de colores como la metodología lo sugiere.

\subsection{Indicadores}

A continuación se muestran los resultados de ICA mediante gráficos y PELN de forma cuantitativa.

\subsubsection{ICA}

Para mostrar un mejor análisis se utilizó el software $\mathrm{R}$ junto con su librería especializada en datos de calidad del aire Openair. Se obtuvieron gráficas que siguen los protocolos establecidos en la metodología de la EPA [16]. De esta forma se pueden distinguir los valores y colores según las categorías definidas en la Tabla II. 
Fig. 3. PANTALLA DE GENERACIÓN DE INDICADORES DE CALIDAD DEL AIRE DESDE LA BODEGA DE DATOS

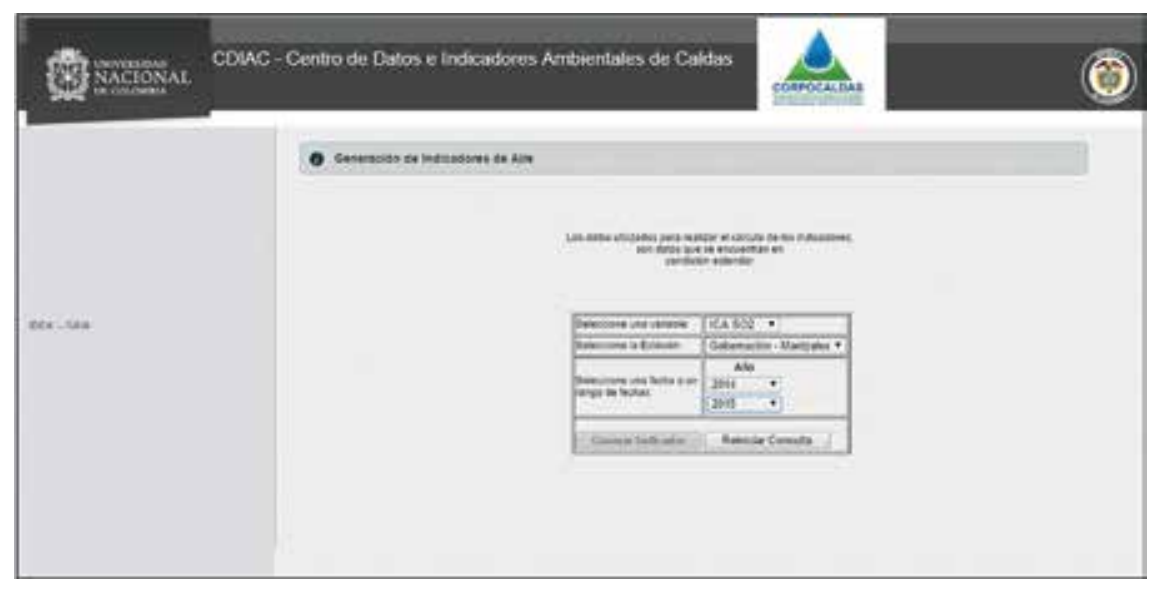

Fuente: autores.

Fig. 4. RESULTADOS NUMÉRICOS DEL ICA CALCULADOS DESDE LA BODEGA DE DATOS

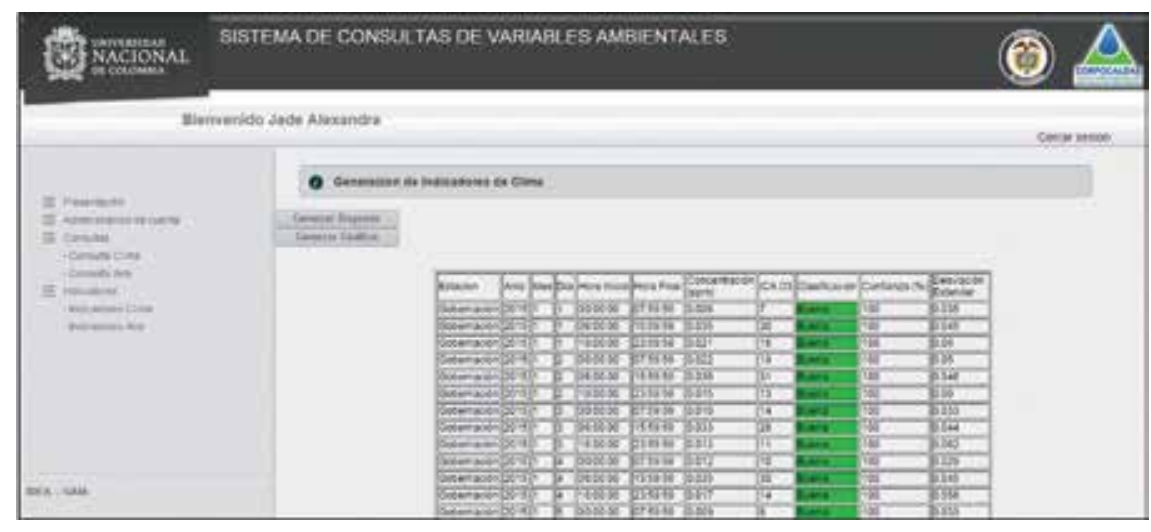

Fuente: autores.

Fig. 5. GRÁFICA DE ICA REALIZADA DESDE LA BODEGA DE DATOS

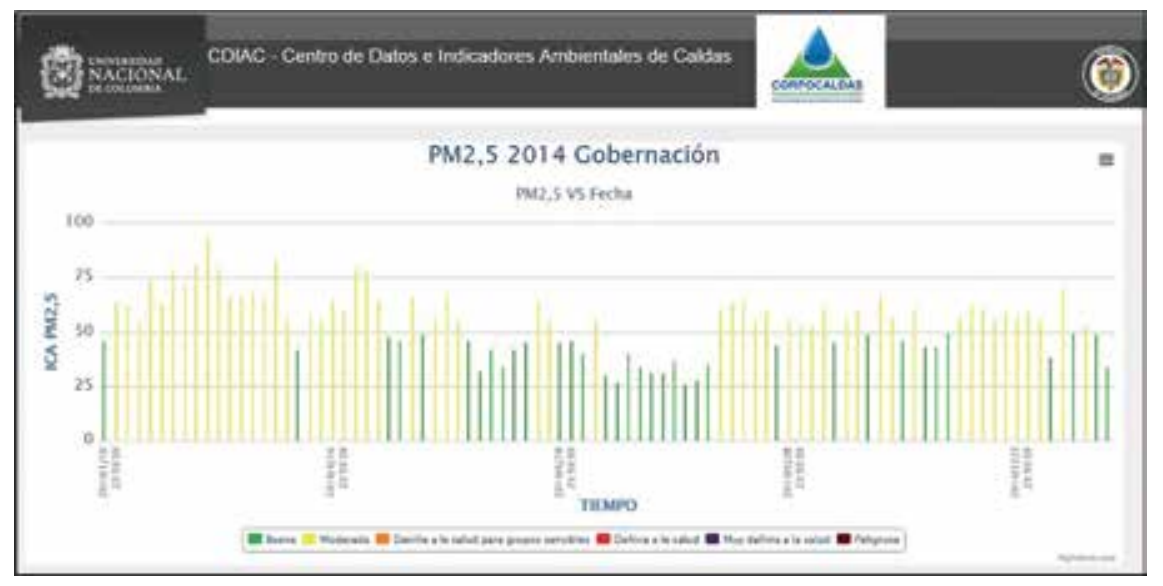

Fuente: autores. 


\section{- PM2.5}

Para representar el material particulado menor a 2.5 micras se realizaron calendarios para todos los años de los que se tienen datos. En la Fig. 6 se muestran los calendarios de los años 2012 y 2014 , como se puede ver, estos cuentan con una barra lateral derecha que constituye los rangos y la escala de colores que define la metodología y muestra en el interior de cada día, el valor correspondiente en ICA.

Los datos resaltados son aquellos que igualan o sobrepasan los límites permisibles de la norma colombiana, en el 2012 este suceso se repite 4 veces entre los meses octubre y septiembre, esto puede asociarse al aumento de la actividad volcánica del Nevado del Ruiz en estas fechas.

Los valores de ICA para $\mathrm{PM}_{2.5}$ en condiciones normales oscilan entre 30 y 60 equivalentes a las clasificaciones Buena y Moderada, respectivamente, estas clasificaciones sugieren que el aire no causa efectos secundarios sobre la salud de la población que lo respira.
- $P M_{10}$

El monitoreo de material particulado menor a 10 micras se realiza en cinco puntos de la ciudad, la Fig. 7 muestra la tendencia del indicador de $\mathrm{PM}_{10}$ en las 5 estaciones junto con los límites que alcanzan el ICA y el límite normado colombiano.

La línea verde que cruza el eje "y" representa la categoría Buena del ICA y la línea roja representa el límite máximo permisible de la ELN.

En la gráfica es notable que la estación Liceo mantiene los valores más altos de ICA alrededor de 40 y que Nubia, Palogrande y Gobernación tienen ICA similares durante toda la tendencia, conservando valores entre 20 y 32 . Esta tendencia puede deberse a que la estación Liceo se encuentra expuesta a mayor flujo vehicular que las otras estaciones. Por otra parte, datos de las estaciones que se encuentran en el Centro Histórico de la ciudad (Gobernación y Liceo) sobrepasan el límite de la clasificación Buena llegando a Moderada.

Fig. 6. CALENDARIOS DE INDICADORES DE CALIDAD DEL AIRE PARA PM 2.5
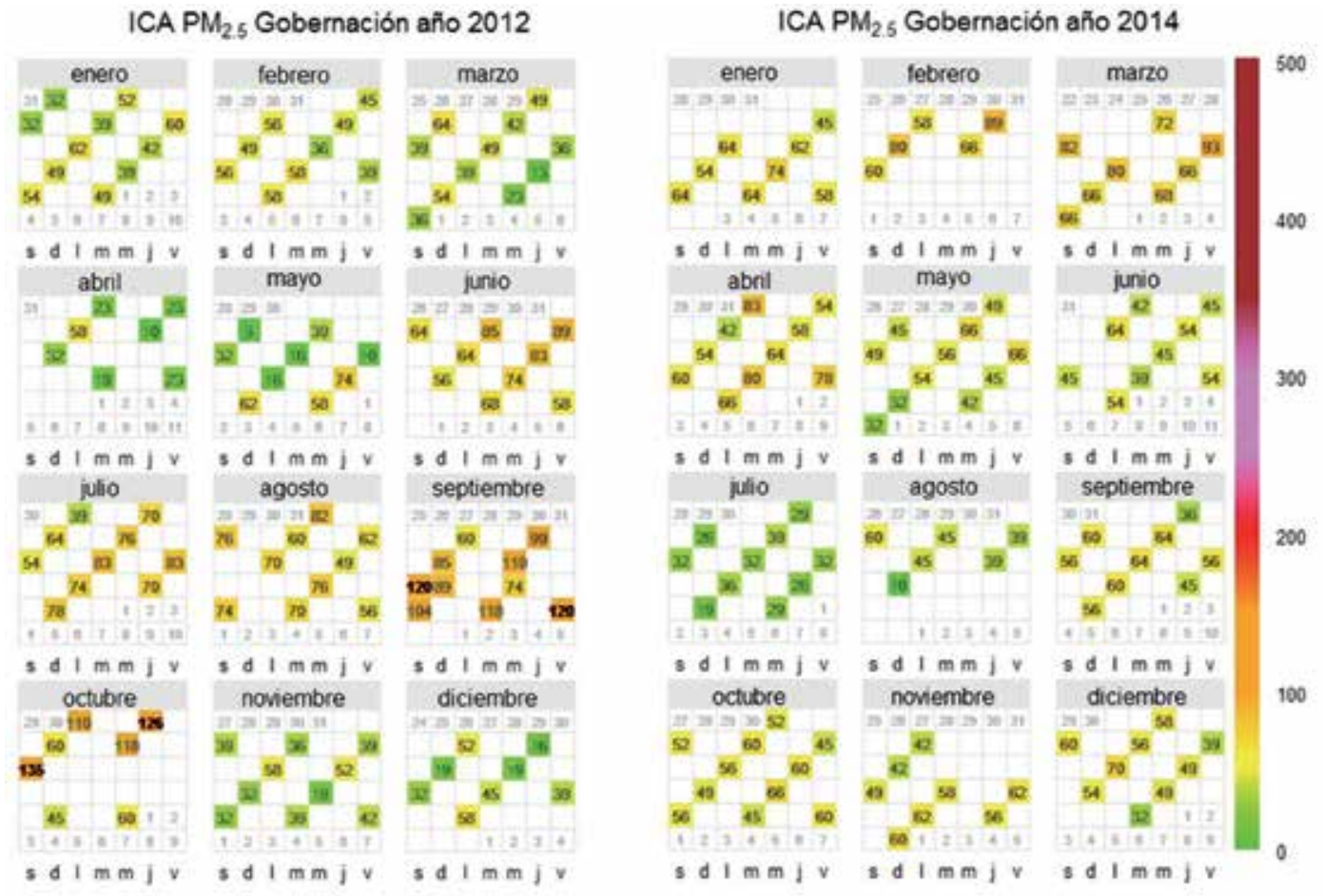

Fuente: autores. 
Fig. 7. TENDENCIA DE ICA PARA PM ${ }_{10}$

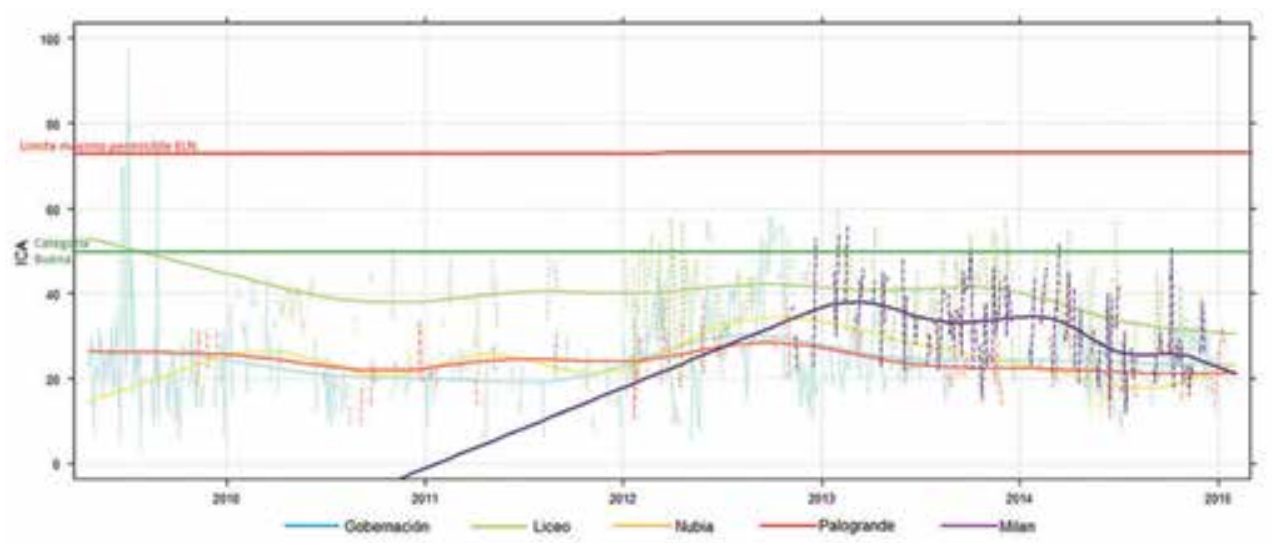

Fuente: autores.

\section{- $\mathrm{O}_{3}$}

Los indicadores de ozono tanto para calcular el ICA como para determinar la excedencia de los límites normados se realizan principalmente con promedios de concentración de ocho horas.

La Fig. 8 representa mediante escalas de colores los valores de ICA, en este caso se ve solo verde, ya que como lo muestra la barra lateral el ICA máximo que alcanza el $\mathrm{O}_{3}$ de aproximadamente 26, es decir, solo hay datos en la categoría "Buena" lo que indica que durante el periodo analizado, la calidad del aire en Manizales es buena. Sin embargo, dentro de esta categoría se distinguen zonas con diferentes valores, siendo mayores aquellos que se visualizan más oscuros.

Se puede observar que en la zona central de la gráfica, es decir, entre las 10:00 y las 15:00 horas del día se concentran los valores más altos. Teniendo en cuenta que el ozono es un contaminante secundario que se forma a partir de reacciones químicas en presencia de luz [17], estos resultados son de esperarse, ya que en este intervalo de tiempo se presenta la mayor radiación solar del día, también las mayores temperaturas que promueven la reactividad en la atmósfera.

Fig. 8. TENDENCIA POR NIVELES DE OCHO HORAS DEL ICA DE $\mathrm{O}_{3}$

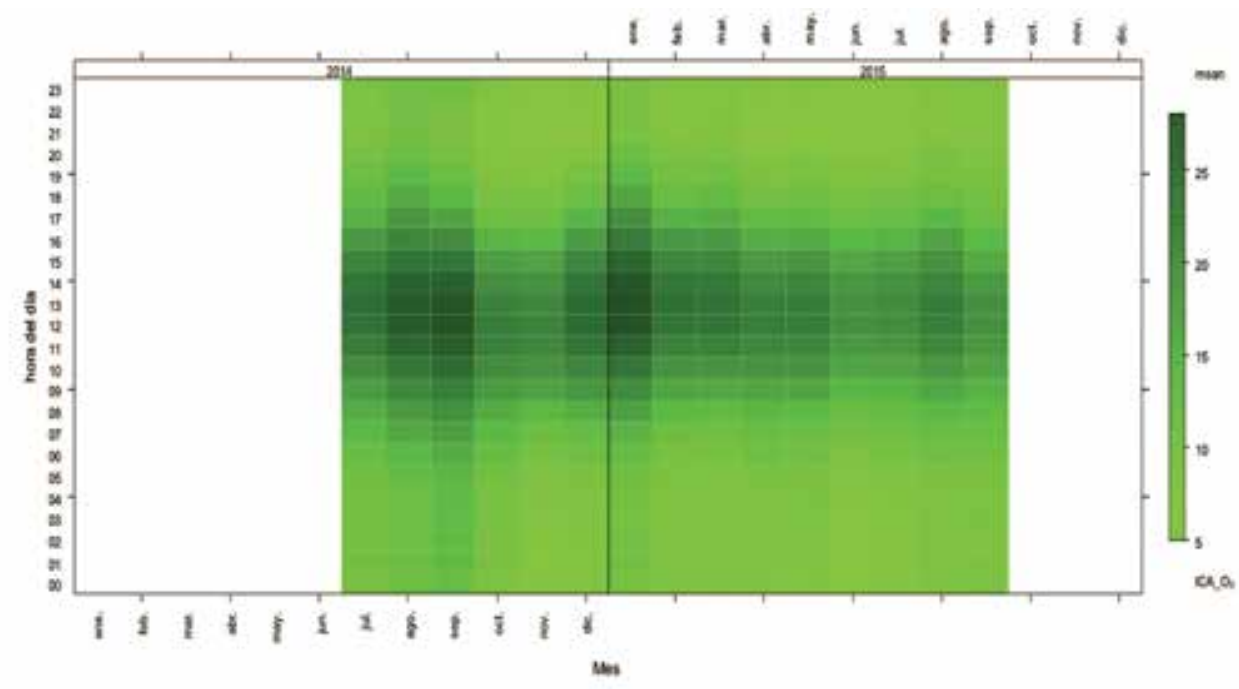




\section{- $\mathrm{SO}_{2}$}

Los ICA para $\mathrm{SO}_{2}$ presentan valores muy bajos como se puede observar en la barra lateral derecha de la Fig. 9 donde las medias móviles oscilan entre 0 y 2.7. Sabiendo esto, se entiende que el ICA permanece en una clasificación Buena, por tal razón toda la gráfica es de color verde. Estos bajos valores pueden verse influenciados por la disminución en la concentración de azufre en el diesel, pasando de 500 ppm a solo 50 ppm a finales del 2012, así como a la implementación del pico y placa para vehículos de transporte público individual y colectivo, a principios del año 2012.

Se realizó la Fig. 9 para analizar mensualmente cómo varía el ICA y así conocer el comportamiento del contaminante, esta muestra claramente que el mayor valor es de 2.7 y se encuentra en el mes de octubre de 2014.

Por otra parte, entre las 16:00 y las 23:00 horas se visualizan las mayores concentraciones del día, estudios de la dirección del viento en

Manizales demuestran que durante esas horas, los vientos provienen desde el Sureste, es decir, desde la ubicación del volcán hacia la ciudad. Lo cual puede explicar las mayores concentraciones de azufre en esas horas.

\subsubsection{PELN}

La Tabla IV muestra los resultados de excedencia de los límites normados. Las estaciones de $\mathrm{PM}_{10}$ de Liceo, Milán, Nubia y Palogrande no mostraron excedencias, solo Gobernación tuvo 2, lo que representa un $0.28 \%$ PM $_{2.5}$ superó el límite 6 veces que equivale a un $1.05 \%$ del total de datos, este es el contaminante con mayor porcentaje de excedencias en Manizales. $\mathrm{O}_{3}$ a las 8 horas es el contaminante que más ha alcanzado en cantidad de veces los niveles perjudiciales para la salud con 22 excedencias, sin embargo, el porcentaje con respecto al total es bajo, dando $0.21 \%$. Los niveles de concentración de $\mathrm{SO}_{2}$ son muy bajos en la ciudad, lo que no ha llegado a representar un riesgo.

Fig. 9. TENDENCIA POR NIVELES DIARIOS DEL ICA DE $\mathrm{SO}_{2}$

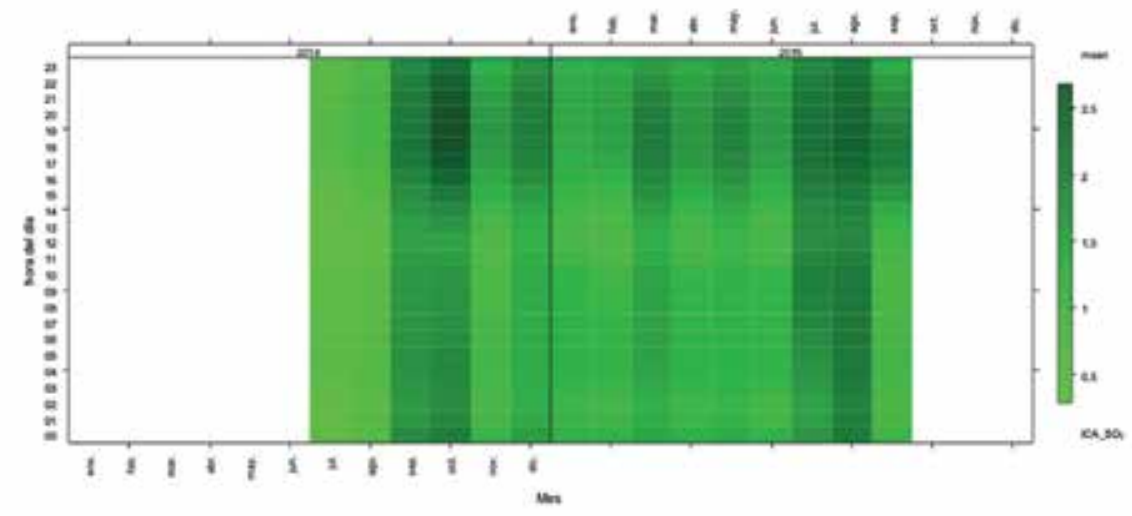

TABLA IV

PORCENTAJE DE EXCEDENCIA DE LOS LÍMITES NORMADOS

\begin{tabular}{|c|c|c|c|c|c|}
\hline & \multicolumn{5}{|c|}{$\mathbf{P M}_{10}$} \\
\hline Estación & Gobernación & Liceo & Milán & Nubia & Palogrande \\
\hline Tiempo de exposición & 24 horas & 24 horas & 24 horas & 24 horas & 24 horas \\
\hline Excedencias & 2 & 0 & 0 & 0 & 0 \\
\hline Registros & 707 & 480 & 256 & 301 & 334 \\
\hline \multirow[t]{2}{*}{ PELN } & $0.28 \%$ & $0.00 \%$ & $0.00 \%$ & $0.00 \%$ & $0.00 \%$ \\
\hline & $\mathrm{PM}_{2.5}$ & \multicolumn{2}{|c|}{$\mathbf{O}_{3}$} & \multicolumn{2}{|c|}{$\mathrm{SO}_{2}$} \\
\hline Estación & Gobernación & Gobernación & Gobernación & Gobernación & Gobernación \\
\hline Tiempo de exposición & 24 horas & 1 hora & 8 horas & 24 horas & Anual \\
\hline Excedencias & 6 & 0 & 22 & 0 & 0 \\
\hline Registros & 572 & 10679 & 10679 & 10195 & 2 \\
\hline PELN & $1.05 \%$ & $0.00 \%$ & $0.21 \%$ & $0.00 \%$ & $0.00 \%$ \\
\hline
\end{tabular}

Fuente: autores 


\section{CONCLUSIONES}

Se implementaron los indicadores de calidad del aire ICA y ELN para los contaminantes $\mathrm{O}_{3}, \mathrm{PM}_{10}$, $\mathrm{PM}_{2.5}$ y $\mathrm{SO}_{2}$. Estos se representaron por medio de diferentes tipos de gráficas realizadas, utilizando el software R y por medio de una bodega de datos que aún se encuentra en construcción.

La bodega de datos es una herramienta útil capaz de almacenar información, y con esta se realizan cálculos y gráficas sistemáticamente. Así disminuye tiempos de procesado y evita errores por manipulación.

Tanto los resultados de indicadores de calidad del aire (ICA) como los de excedencia de los límites normados suministran información que ayuda a comprender la calidad del aire en la zona estudiada de manera fácil y contundente.

Los indicadores para $\mathrm{PM}_{2.5}$ muestran que la calidad del aire en Manizales se ha visto afectada en ocasiones por la actividad del volcán Nevado del Ruiz, unos eventos muy evidentes se presentaron en los meses de agosto y septiembre de 2012, que llevaron a sobrepasar cuatro días los niveles máximos permisibles por la legislación. Aunque estos eventos del volcán afectan la calidad del aire en Manizales con respecto a $\mathrm{PM}_{2.5}$ en condiciones normales, se presentan ICA entre 30 y 60 que no causan efectos negativos para la salud de la población.

Los indicadores de $\mathrm{PM}_{10}$ revelan que las concentraciones de cada estación no varían significativamente. Sin embargo, la estación Liceo es la que tiene los ICA más altos, esto es factible, ya que esta es la estación con mayor concurrencia de tráfico vehicular.

El análisis de $\mathrm{O}_{3}$ cada ocho horas demuestra que las mayores concentraciones se presentan entre las 10:00 y las 15:00 horas donde la radiación solar es mayor, lo cual es de esperarse, ya que este es un contaminante secundario que se forma por reacciones fotoquímicas.

\section{AGRADECIMIENTOS}

Proyecto: "Implementación de indicadores de línea base ambiental y construcción de una bodega de datos para apoyar la generación y análisis de los mismos como parte de la Fase III del proyecto de estructuración de la Línea Base Ambiental del
Departamento de Caldas", Corporación Autónoma de Caldas, CORPOCALDAS - Universidad Nacional de Colombia Sede Manizales

\section{REFERENCIAS}

[1] CONPES, "Lineamientos para la formulación de la politica de prevencion y control de la contaminación del aire," Bogota D. C, Colombia, 2005.

[2] INEEC and SEMARNAT, "Contaminantes criterio," 2013. [Online]. Available: http://www.inecc.gob.mx/ calaire-indicadores/523-calaire-cont-criterio.

[3] E. Sánchez, K. Ahmed, and Y. Awe, "Prioridades ambientales para la reducción de la pobreza en Colombia. Un análisis ambiental del país para Colombia," Bogota D. C: The International Bank for Reconstruction and Development/The World Bank, 2006.

[4] M. Jaramillo, D. E. González, and M. E. Núñez, “Índice integrado de calidad del aire para ciudades colombianas," Rev. Fac. Ing. Univ. Antioquia, vol. 48, pp. 97106, 2009.

[5] UN, "Centro de Datos e Indicadores Ambientales de Caldas," Generador de indicadores climatológicos, 2015. [Online]. Available: http://cdiac.manizales.unal. edu.co/IDEA/resena.php. [Accessed: 22-Jun-2016].

[6] S.-T. Li and L.-Y. Shue, "Data mining to aid policy making in air pollution management," Expert Syst. Appl., vol. 27, no. 3, pp. 331-340, Oct. 2004.

[7] UN and Corpocaldas, "CDIAC - Centro de Datos e Indicadores Ambientales de Caldas," Generador de indicadores climatológicos, 2015. [Online]. Available: http://froac.manizales.unal.edu.co/cube/IDEA/index. php. [Accessed: 04-Sep-2015].

[8] J. Li, Á. Pérez, N. Duque, and B. Aristizábal, “V Congreso Colombiano y Conferencia Internacional de Calidad del Aire y Salud Pública II CMAS SOUTH AMERICAN CONFERENCE," in Indicadores de calidad del aire en Manizales: Implemetación y representación, Bucaramanga, 2015, pp. 24-36.

[9] P. Kassomenos, a. N. Skouloudis, S. Lykoudis, and $\mathrm{H}$. a. Flocas, “'Air-quality indicators' for uniform indexing of atmospheric pollution over large metropolitan areas," Atmos. Environ., vol. 33, no. 12, pp. 18611879, Jun. 1999.

[10] DANE, "Proyeccion Municipios de Colombia 20052020," Colombia, 2005.

[11] N. Franco, "Encuesta de Percepción Ciudadana Manizales Cómo Vamos," 2014. [Online]. Available: www. manizalescomovamos.org. 
[12] MAVDT, Manual de diseño de sistemas de vigilancia de la calidad del aire, 2010, p. 137.

[13] OPS/OMS, Protocolo para la vigilancia sanitaria y ambiental de los efectos en salud relacionados con la contaminación del aire en Colombia. Colombia, 2012, pp. 171-175.

[14] MAVDT, Resolución 610, no. 610. Colombia: Resolución 0601 del 4 de abril del 2006, pp. 1-8.

[15] IDEAM, "Documento soporte norma calidad del aire," Ley 610 del 2010, Bogotá, Colombia, 2005.
[16] J. Clench-Aas, C. Guerreiro, and A. Bartonova, Air quality indicators. Kjeller- Norway: Norsk institutt for luftforskning, 1998.

[17] D. W. Purves, J. P. Caspersen, P. R. Moorcroft, G. C. Hurtt, and S. W. Pacala, "Human-induced changes in US biogenic volatile organic compound emissions: evidence from long-term forest inventory data," Glob. Chang. Biol., vol. 10, no. 10, pp. 1737-1755, Oct. 2004. 